\title{
Probability of local liquefaction of saturated sands in half-space domains under a train of surface point explosions
}

\author{
Luis A. de Béjar* \\ Geotechnical and Structures Laboratory, US Army Engineer Research and Development Center, ATTN: \\ CEERD-GS-M (Bldg. 5008), 3909 Halls Ferry Road, Vicksburg, MS 39180-6199, USA
}

Received 1 October 2007

Revised 13 January 2009

\begin{abstract}
The probability of liquefaction in saturated sand deposits subjected to a train of shear stress pulses propagating from a point blast source applied at the boundary surface of the elastic medium is investigated. The load effect is evaluated in approximate closed form using three-dimensional tensorial mathematical physics in polar cylindrical coordinates. The adopted criterion of liquefaction has been experimentally verified both in the laboratory and in the field when continua of saturated sands were subjected to equivalent cyclic shear stress due to earthquake excitation. A first-order second-moment technique for the probabilistic assessment of the liquefaction potential in practical situations is developed and implemented in a computer program. Parametric studies are conducted to examine the sensitivity of results to the second-moment characterization of intervening key physical quantities.
\end{abstract}

\section{Introduction}

Experimental evidence shows that saturated sands are susceptible to liquefaction when subjected to a combination of pore pressure increase and alternating shear forces from stress waves emanating from surface blast sources [2-6]. Liquefaction occurs when pore water pressure rises to a level such that there is a complete loss of inter-granular contact and, therefore, of effective stress within the soil skeleton.

To allow mathematical tractability of the problem in this investigation, the intervening physical components are simplified. The load effect of a concentrated explosion is idealized by means of a single compressive load pulse spatially concentrated and temporally exponential. The soil is considered as a homogeneous and isotropic linearelastic continuum. And the effect of existing, transient, and residual pore pressures are taken into account through an empirical criterion for the liquefaction of saturated non-cohesive soils under repeated cycles of shear distortion. Notice that the blast compressive pulse induces in the medium propagating waves of both dilatational and distortional nature, and that both contribute to the shear stress response in the half-space field. Consequently, both dilatational and distortional stress waves propagate from the explosion source with distinct velocities $c_{1}$ and $c_{2}$, respectively.

The governing equations of motion are conveniently formulated in terms of scalar and vector potentials in cylindrical polar coordinates in the time domain $[10,14]$. The formulation is then sequentially transformed into the Laplace and Hankel domains, and the solutions for the potentials are obtained in Hankel space. The inversion of the response is carried out approximately. Bessel functions within the resulting mathematical expressions are expanded in Maclaurin series and subsequently approximated by truncation, on account of the small magnitude of

\footnotetext{
*Corresponding author. Tel.: +1 601634 3762; Fax: +1 601634 2309; E-mail: Luis.A.DeBejar@erdc.usace.army.mil.
} 


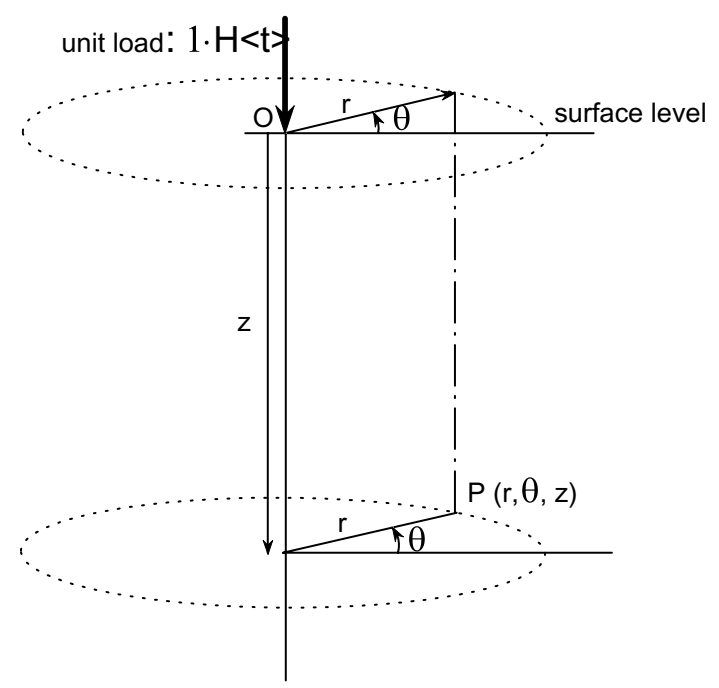

Fig. 1. Unit-step-function load normal to the horizontal surface plane of a half-space linear elastic domain, acting at a source point $\mathrm{O}$ on the surface, and an arbitrary field point $\mathrm{P}$, described in cylindrical coordinates.

the arguments, as compared to unity. The response is obtained in the time domain through manipulation of transform integrals.

The indicial admittance function is obtained first, i.e., the response to a load of unit magnitude that remains constant in time [10]. Then, the unit-impulse response function is obtained from the time rate of variation of the indicial admittance function. The response to a general forcing function is expressed in the time domain using the corresponding convolution with the unit-impulse response function [7,15]. Mathematical expressions are reduced for calculations by evaluating the shear stress transmitted to the point under consideration at the time when it is considered to be a maximum. Since almost all the blast energy is transmitted to the elastic half-space domain in the form of direct compression, it is reasoned that the maximum stress effect at critical locations is attained at the time of first arrival of the dilatational waves. In fact, substantial shear stresses act on planes rotated in space with respect to the local principal directions.

The adopted criterion for liquefaction has been experimentally verified both in the laboratory and in the field when continua of saturated sands were subjected to equivalent cyclic shear stress due to earthquake excitation [12,16].

Models for the probability of liquefaction are formulated using two separate approaches. In the first approach, both the load effect and the resistance are postulated as log-normally distributed. In this case, an exact expression for the probability of surviving liquefaction is obtained [8]. In the second approach, a more realistic condition of uncertainty is assumed and a first-order second-moment formulation for the probability of liquefaction is proposed. Load effect and resistance are both characterized by the corresponding mean and coefficient of variation. The limit state for liquefaction is linearized about the mean vector of basic random variables, and the reliability index $\beta$ is obtained approximately [1]. In this case, the probability of liquefaction is rigorously correct only if the load effect and the resistance are both normally distributed. Otherwise, the probability of liquefaction calculated on the basis of the Gaussian assumption is still an approximate measure of the chances of liquefaction [9].

The second approach was implemented in a computer program for practical applications of the model in the field, and a parametric study on the variation of the probability of liquefaction with the second-moment parameters of the blasting load was conducted.

\section{Load effect}

\subsection{Stress wave propagation due to normal loading on a half-space domain}

The origin of coordinates is located at the point of application of the charge (Fig. 1). In this particular problem, it is convenient to define cylindrical polar coordinates specified by the triplet $(r, \theta, z)$, with $z \geqslant 0$. On account of the 
axi-symmetry of the problem, physical quantities in the formulation are independent of $\theta$, and the transverse polar displacement itself $\left(u_{\theta}\right)$ is zero. Therefore, the displacement vector $\mathbf{u}$ is given by:

$$
\mathbf{u}(r, z, t)=u_{r}(t) \cdot \mathbf{e}_{r}+u_{z}(t) \cdot \mathbf{e}_{z} .
$$

This displacement field may be expressed as the summation of irrotational and solenoidal component fields, respectively, or in terms of the corresponding potentials as [10]:

$$
\mathbf{u}=\nabla \phi+\nabla \times \mathbf{H},
$$

in which the vector potential $\mathbf{H}$ itself is solenoidal (a zero-divergence vector), i.e.,

$\nabla \cdot \mathbf{H}=0$. Notice that the curl of $\mathbf{H}$ in Eq. (2) is given by [11]:

$$
\begin{aligned}
\nabla \times \mathbf{H} & =\frac{1}{r} \cdot\left|\begin{array}{ccc}
e_{r} & r \cdot \mathbf{e}_{\theta} & \mathbf{e}_{z} \\
\frac{\partial}{\partial r} & \frac{\partial}{\partial \theta} & \frac{\partial}{\partial z} \\
H_{r} & r \cdot H_{\theta} & H_{z}
\end{array}\right| \\
& =\frac{1}{r} \cdot \frac{\partial}{\partial r}\left(r \cdot H_{\theta}\right) \cdot \mathbf{e}_{z}-\frac{\partial H_{\theta}}{\partial z} \cdot \mathbf{e}_{r},
\end{aligned}
$$

since the components $H_{r}$ and $H_{z}$ do not depend on $\theta$. Consequently, Eq. (2) may be written equivalently as:

$$
\mathbf{u}=\nabla \phi+\nabla \times\left(H_{\theta} \cdot \mathbf{e}_{\theta}\right) .
$$

The governing equations for the scalar and vector potentials ( $\phi$ and $\mathbf{H})$ are given by the corresponding scalar and vector wave equations, respectively:

$$
\nabla^{2} \phi=\frac{1}{c_{1}^{2}} \cdot \ddot{\phi}
$$

and

$$
\begin{gathered}
\left(\nabla^{2} \mathbf{H}\right)_{\theta}=\frac{1}{c_{2}^{2}} \cdot(\ddot{\mathbf{H}})_{\theta} \\
\text { or } \quad \nabla^{2} H_{\theta}-\frac{1}{r^{2}} \cdot H_{\theta}=\frac{1}{c_{2}^{2}} \cdot \ddot{H}_{\theta},
\end{gathered}
$$

where $\nabla^{2}=$ the Laplacian operator in cylindrical polar coordinates:

$$
\nabla^{2}=\frac{1}{r} \cdot \frac{\partial}{\partial r}\left(r \cdot \frac{\partial}{\partial r}\right)+\frac{\partial^{2}}{\partial z^{2}}
$$

and $c_{1}, c_{2}=$ the velocity of propagation of dilatational and distortional waves in the elastic medium, respectively.

Equation (5b) can be transformed into the wave equation by defining a smooth function $\psi$ such that:

$$
H_{\theta}=-\frac{\partial \psi}{\partial r}
$$

then, Eq. (5b) becomes:

$$
\nabla^{2} \psi=\frac{1}{c_{2}^{2}} \cdot \ddot{\psi}
$$

\subsubsection{Displacements}

With the gradient operator in cylindrical polar coordinates given by

$$
\nabla=u_{r} \frac{\partial}{\partial r}+u_{z} \frac{\partial}{\partial z}
$$

the displacement components are obtained as [10]:

$$
u_{r}=\frac{\partial \phi}{\partial r}-\frac{\partial H_{\theta}}{\partial z}=\frac{\partial \phi}{\partial r}+\frac{\partial^{2} \psi}{\partial z \partial r}
$$




$$
u_{z}=\frac{\partial \phi}{\partial z}+\frac{1}{r} \cdot \frac{\partial}{\partial r}\left(r \cdot H_{\theta}\right)=\frac{\partial \phi}{\partial z}-\frac{1}{r} \cdot\left[r \cdot \frac{\partial^{2} \psi}{\partial r^{2}}+\frac{\partial \psi}{\partial r}\right]
$$

However, from Eq. (8) one has:

$$
\frac{1}{r} \cdot \frac{\partial}{\partial r}\left(r \cdot \frac{\partial \psi}{\partial r}\right)=\frac{1}{c_{2}^{2}} \cdot \ddot{\psi}-\frac{\partial^{2} \psi}{\partial z^{2}}
$$

and inserting Eq. (10c) into Eq. (10b), one gets:

$$
u_{z}=\frac{\partial \phi}{\partial z}+\frac{\partial^{2} \psi}{\partial z^{2}}-\frac{1}{c_{2}^{2}} \cdot \ddot{\psi}
$$

Naturally, one also has:

$$
u_{\theta}=0 \text {. }
$$

\subsubsection{Stresses}

The material constitutive relations for the elastic medium render the expressions for the components of the stress tensor in cylindrical polar coordinates:

$$
\begin{aligned}
& \tau_{r r}=\lambda \cdot \nabla^{2} \phi+2 \mu \cdot\left(\frac{\partial^{2} \phi}{\partial^{2} r}+\frac{\partial^{3} \psi}{\partial^{2} r \partial z}\right) \\
& \tau_{\theta \theta}=\lambda \cdot \nabla^{2} \phi+\frac{2 \mu}{r} \cdot\left(\frac{\partial \phi}{\partial r}+\frac{\partial^{2} \psi}{\partial r \partial z}\right) \\
& \tau_{z z}=\lambda \cdot \nabla^{2} \phi+2 \mu \cdot \frac{\partial}{\partial z}\left(\frac{\partial \phi}{\partial z}+\frac{\partial^{2} \psi}{\partial^{2} z}-\frac{1}{c_{2}^{2}} \cdot \ddot{\psi}\right) \\
& \tau_{r z}=\mu \cdot \frac{\partial}{\partial r}\left(2 \frac{\partial \phi}{\partial z}+2 \frac{\partial^{2} \psi}{\partial^{2} z}-\frac{1}{c_{2}^{2}} \cdot \ddot{\psi}\right) \\
& \tau_{\theta z}=\tau_{r \theta}=0,
\end{aligned}
$$

where $(\lambda, \mu)=$ the Lamé elastic constants for the medium.

\subsubsection{Boundary and initial conditions}

The stress boundary conditions refer to the stress-free condition of the surface, except for the singularity at the point of application of the concentrated dynamic load. Expressed mathematically, one has at the surface $(z=0)$ :

$$
\begin{aligned}
\tau_{z r} & =0 \\
\tau_{z z} & =F(r) \cdot H\langle t\rangle,
\end{aligned}
$$

where $F(r)$ is the spatial variation of the externally applied surface stress, and $\mathrm{H}<t>$ is the Heaviside unit-step function, which is the time variation corresponding to a suddenly applied constant pressure. Notice that the spatial variation $F(r)$ may be expressed as a Fourier-Bessel integral:

$$
F(r)=\int_{0}^{\infty} \xi \cdot J_{0}(\xi \cdot r) \cdot\left\{\int_{0}^{\infty} F(\sigma) \cdot \sigma \cdot J_{0}(\sigma \cdot \xi) \cdot d \sigma\right\} \cdot d \xi
$$

In our problem, $F(r)$ corresponds to a point load:

$$
F(r)=\frac{\delta(r)}{2 \pi r},
$$


where $\delta(r)=$ the Dirac delta function applied at the origin of coordinates, such that the magnitude of the total applied force is:

$$
\int_{\Omega} F(r) \cdot d A=\int_{0}^{2 \pi} d \theta \int_{0}^{\infty} \frac{\delta(r)}{2 \pi r} \cdot r d r=1,
$$

where $\Omega=$ the area of the medium boundary surface. Therefore Eq. (13) gives the following Fourier-Bessel integral for the spatial variation of the loading:

$$
F(r)=\frac{1}{2 \pi} \cdot \int_{0}^{\infty} \xi \cdot J_{0}(\xi \cdot r) d \xi .
$$

The system is assumed to be initially at rest (zero initial conditions).

\subsection{Transformed equations}

Let $L^{*}$ be the Laplace transform operator. Then the Laplace transform of displacements and stresses in the governing equations will be denoted by the corresponding capital letters as:

$$
\begin{aligned}
U_{i}(r, z, p) & \equiv L^{*}\left(u_{i}(r, z, t)\right) \\
T_{i j}(r, z, p) & \equiv L^{*}\left(\tau_{i j}(r, z, t)\right),
\end{aligned}
$$

where $\mathrm{p}$ is the Laplace variable. Likewise, the Laplace transform of the potential functions will be denoted as:

$$
\tilde{\phi} \equiv L^{*}(\phi) \quad \text { and } \quad \tilde{\psi} \equiv L^{*}(\psi)
$$

The Laplace transform of the governing wave Eqs (5a) and (8) become:

$$
\nabla^{2} \tilde{\phi}-\frac{1}{c_{1}^{2}} \cdot p^{2} \cdot \tilde{\phi}=0
$$

and

$$
\nabla^{2} \tilde{\psi}-\frac{1}{c_{2}^{2}} \cdot p^{2} \cdot \tilde{\psi}=0
$$

respectively.

The Laplace transform of the displacement Eqs (10a) and (10d) are:

$$
U_{r}=\frac{\partial \tilde{\phi}}{\partial r}+\frac{\partial^{2} \tilde{\psi}}{\partial z \partial r}
$$

and

$$
U_{z}=\frac{\partial \tilde{\phi}}{\partial z}+\frac{\partial^{2} \tilde{\psi}}{\partial^{2} z}-\frac{1}{c_{2}^{2}} \cdot p^{2} \cdot \tilde{\psi}
$$

The Laplace transform of the stress Eqs (11a)-(11d) may be obtained as:

$$
\begin{aligned}
& T_{r r}=\lambda \cdot \nabla^{2} \tilde{\phi}+2 \mu \cdot\left(\frac{\partial^{2} \tilde{\phi}}{\partial r^{2}}+\frac{\partial^{3} \tilde{\psi}}{\partial r^{2} \partial z}\right) \\
& T_{\theta \theta}=\lambda \cdot \nabla^{2} \tilde{\phi}+\frac{2 \mu}{r} \cdot\left(\frac{\partial \tilde{\phi}}{\partial r}+\frac{\partial^{2} \tilde{\psi}}{\partial r \partial z}\right) \\
& T_{z z}=\lambda \cdot \nabla^{2} \tilde{\phi}+2 \mu \cdot\left(\frac{\partial^{2} \tilde{\phi}}{\partial z^{2}}+\frac{\partial^{3} \tilde{\psi}}{\partial^{3} z}-\frac{1}{c_{2}^{2}} \cdot p^{2} \cdot \frac{\partial \tilde{\psi}}{\partial z}\right)
\end{aligned}
$$




$$
T_{r z}=\mu \cdot\left(2 \cdot \frac{\partial^{2} \tilde{\phi}}{\partial r \partial z}+2 \cdot \frac{\partial^{3} \tilde{\psi}}{\partial z^{2} \partial r}-\frac{1}{c_{2}^{2}} \cdot p^{2} \cdot \frac{\partial \tilde{\psi}}{\partial r}\right)
$$

and the Laplace transform of the boundary conditions (12a)-(12b) on the surface $(z=0)$ are:

$$
\begin{aligned}
T_{z r} & =0 \\
T_{z z} & =F(r) \cdot \frac{1}{p}, \quad \text { at } z=0 .
\end{aligned}
$$

In this model, the parameter of response controlling liquefaction in the elastic medium is the oscillating shear stress given by Eq. (11d), with Laplace transform given by Eq. (21d) subjected to the boundary conditions stipulated by Eqs (22a) and (22b). In order to solve these equations, the formulation will be transformed next from the Laplace to the Hankel domain.

The Hankel transform of order $\nu$ of a general function $f(r)$, with nonnegative $r$, is defined as:

$$
\int_{0}^{\infty} r \cdot J_{\nu}(\xi \cdot r) \cdot f(r) d r
$$

where $J_{\nu}$ ( ) is the Bessel function of order $\nu$, and $\xi$ is the Hankel variable. Hankel transforms of functions of order zero and one will be denoted as:

$$
\bar{f}(\xi) \text { and } \hat{f}(\xi)
$$

respectively.

The Hankel transform of order $\nu$ of the derivative $d f / d r$ is given by:

$$
\int_{0}^{\infty} r \cdot J_{\nu}(\xi \cdot r) \cdot \frac{d f(r)}{d r} d r .
$$

Applying integration by parts to Eq. (23b) and using the recursion formula for Bessel functions, one obtains:

$$
\frac{d \hat{f}}{d r}(\xi)=-\xi \cdot \bar{f}(\xi)
$$

Applying the Hankel transform of order one to Eq. (21d), and considering Eq. (24), the following expression results for the transformed shear stress in Hankel space:

$$
\hat{T}_{r z}=-\xi \cdot \mu \cdot\left(2 \bar{\phi}^{\prime}+2 \bar{\psi} "-k^{2} \cdot \bar{\psi}\right)
$$

where $k^{2}=p^{2} / c_{2}^{2}$, and a prime superscript (') means $\partial / \partial z$. Similarly, transforming into Hankel space the boundary conditions Eqs (22a)-(22b), one gets:

$$
2 \bar{\phi}^{\prime}+2 \bar{\psi} "-k^{2} \cdot \bar{\psi}=0, \quad \text { at } z=0
$$

and

$$
\lambda \cdot h^{2} \cdot \bar{\phi}+2 \mu \cdot\left(\bar{\phi}^{\prime \prime}+\bar{\psi}^{\prime \prime \prime}-k^{2} \cdot \bar{\psi}^{\prime}\right)=\frac{1}{p} \cdot \frac{1}{2 \pi}, \quad \text { at } z=0,
$$

where $h^{2}=p^{2} / c_{1}^{2}$.

Using Eqs (26a)-(26b), Eq. (25) gives the solution for the shear stress field in Hankel space:

$$
\hat{T}_{r z}(\xi, z, p)=-\frac{1}{\pi} \cdot \frac{1}{p} \cdot(\alpha \cdot \xi) \cdot\left\{-\frac{k^{2}+2 \xi^{2}}{D} \cdot e^{-\alpha \cdot z}+\frac{2 \beta^{2}-k^{2}}{D} \cdot e^{-\beta \cdot z}\right\},
$$

where

$$
\alpha^{2}=\xi^{2}+\frac{p^{2}}{c_{1}^{2}}
$$




$$
\beta^{2}=\xi^{2}+\frac{p^{2}}{c_{2}^{2}}
$$

and

$$
D=\left(k^{2}+2 \xi^{2}\right)^{2}-4 \alpha \cdot \beta \cdot \xi^{2}
$$

\subsection{Indicial admittance function}

Transforming Eq. (27a) from Hankel space back into the Laplace domain, one writes:

$$
T_{r z}(r, z, p)=\frac{1}{\pi \cdot p} \int_{0}^{\infty} \alpha \cdot \xi^{2} \cdot \frac{\left(k^{2}+2 \xi^{2}\right) \cdot e^{-\alpha \cdot z}-\left(2 \beta^{2}-k^{2}\right) \cdot e^{-\beta \cdot z}}{D} \cdot J_{1}(\xi \cdot r) d \xi .
$$

In order to evaluate the integral in Eq. (28), let us define two improper integrals:

$$
\begin{aligned}
& I_{1}=\frac{1}{p} \cdot \int_{0}^{\infty} \frac{\alpha \cdot \xi^{2} \cdot\left(k^{2}+2 \xi^{2}\right) \cdot e^{-\alpha \cdot z}}{\left(2 \xi^{2}+k^{2}\right)^{2}-4 \alpha \cdot \beta \cdot \xi^{2}} \cdot J_{1}(\xi \cdot r) d \xi \\
& I_{2}=-\frac{1}{p} \cdot \int_{0}^{\infty} \frac{\alpha \cdot \xi^{2} \cdot\left(2 \beta^{2}-k^{2}\right) \cdot e^{-\beta \cdot z}}{\left(2 \xi^{2}+k^{2}\right)^{2}-4 \alpha \cdot \beta \cdot \xi^{2}} \cdot J_{1}(\xi \cdot r) d \xi
\end{aligned}
$$

Let us consider $I_{1}$ first. By virtue of Eq. (27b), for a fixed value of the Laplace variable $p$, the Hankel variable $\xi$ may be regarded as a function of $\alpha$. And a change of variables defined by the transformation $\alpha \cdot z=p \cdot t$ maps integral $I_{1}$ into the time domain:

$$
I_{1}=\frac{p}{z \cdot c_{2}} \cdot \int_{0}^{\infty} \frac{\tau^{2} \cdot \sqrt{\tau^{2}-\varepsilon} \cdot\left(2 \tau^{2}+\delta_{2}\right) \cdot J_{1}\left(k \cdot r \cdot \sqrt{\tau^{2}-\varepsilon}\right) \cdot H\left\langle t-\frac{z}{c_{1}}\right\rangle}{\left(2 \tau^{2}+\delta_{2}\right)^{2}-4 \tau \cdot \sqrt{\tau^{2}+\delta_{1}} \cdot\left(\tau^{2}-\varepsilon\right)} \cdot e^{-p \cdot t} d t
$$

where $\tau=\left(c_{2} / z\right) \cdot t, \varepsilon=\left(c_{2} / c_{1}\right)^{2}, \delta_{1}=1-\varepsilon, \delta_{2}=1-2 \varepsilon, \mathrm{H}<>$ is the Heaviside unit-step function, and in which the following relations have been used:

$$
\begin{aligned}
\alpha & =k \cdot \tau \\
\xi^{2} & =k^{2} \cdot\left(\tau^{2}-\varepsilon\right) \\
2 \xi^{2}+k^{2} & =k^{2} \cdot\left(2 \tau^{2}+\delta_{2}\right) \\
\beta^{2} & =k^{2} \cdot\left(\tau^{2}+\delta_{1}\right)
\end{aligned}
$$

Therefore, integral $I_{1}$ may be expressed concisely as:

$$
I_{1}=\frac{p}{z \cdot c_{2}} \cdot \int_{0}^{\infty} \varphi(\tau) \cdot J_{1}\left(k \cdot r \cdot \sqrt{\tau^{2}-\varepsilon}\right) \cdot H\left\langle t_{1}\right\rangle \cdot e^{-p \cdot t} d t
$$

with

$$
\varphi(\tau) \equiv \frac{\tau^{2} \cdot \sqrt{\tau^{2}-\varepsilon} \cdot\left(2 \tau^{2}+\delta_{2}\right)}{\left(2 \tau^{2}+\delta_{2}\right)^{2}-4 \tau \cdot \sqrt{\tau^{2}+\delta_{1}} \cdot\left(\tau^{2}-\varepsilon\right)}
$$

and $t_{1}=t-z / c_{1}$.

Now let's examine the argument of the Bessel function $J_{1}$ in Eq. (30c). Since almost all the blast energy is transmitted to the elastic half-space domain in the form of direct compression, it is reasoned that the maximum stress effect at critical locations is attained at the time of first arrival of the dilatational waves. Substantial shear stresses are obtained then upon evaluation of the state of stress on planes rotated in space with respect to the local radial 
direction. For a given radial location $(r)$ under consideration, the critical time for evaluation of the shear stress is then shortly after the first arrival of the dilatational wave. After that time, the amplitude of the local history of response decays progressively (the elasticity of the medium introduces effective damping in the system [10]). Therefore, the quantity represented by the radical in the argument $\left(\sqrt{ }\left(\tau^{2}-\varepsilon\right)\right.$ ) may be expressed approximately as:

$$
\sqrt{\left(\tau^{2}-\varepsilon\right)} \approx 2 \sqrt{\varepsilon} \cdot\left(1+\frac{\Delta t}{4 z / c_{1}}\right) \cdot \sqrt{\frac{\Delta t}{2 z / c_{1}}},
$$

where $\Delta \mathrm{t}$ is a small time increment, as compared to the arrival time $z / c_{1}$. Therefore, the radical is of small magnitude and the Bessel function $J_{1}$ may be approximated by its truncated infinite series expansion, i.e.:

$$
J_{1}(x) \approx \frac{x}{2}-\frac{x^{3}}{16}
$$

Consequently, integral $I_{1}$ can now be expressed as:

$$
I_{1}=\frac{p}{2 z \cdot c_{2}} \cdot \int_{0}^{\infty} \varphi(\tau) \cdot\left(k \cdot r \cdot \sqrt{\tau^{2}-\varepsilon}-\left(\frac{k \cdot r}{2}\right)^{3} \cdot\left(\tau^{2}-\varepsilon\right)^{3 / 2}\right) \cdot H\left\langle t_{1}\right\rangle \cdot e^{-p \cdot t} d t
$$

or

$$
I_{1}=\frac{1}{2 z \cdot c_{2}} \cdot\left\{\begin{array}{l}
\frac{r}{c_{2}} \cdot p^{2} \cdot \int_{0}^{\infty} \varphi(\tau) \cdot \sqrt{\tau^{2}-\varepsilon} \cdot H\left\langle t_{1}\right\rangle \cdot e^{-p \cdot t} d t+ \\
-\left(\frac{r}{2 c_{2}}\right)^{3} \cdot p^{4} \cdot \int_{0}^{\infty} \varphi(\tau) \cdot\left(\tau^{2}-\varepsilon\right)^{3 / 2} \cdot H\left\langle t_{1}\right\rangle \cdot e^{-p \cdot t} d t
\end{array}\right\}
$$

On account of the zero initial conditions of the problem, the quantities within the braces in Eq. (34) are precisely expressions for the Laplace transform of some derivatives. Therefore, integral $I_{1}$, being a linear combination of Laplace transforms, must be itself a Laplace transform of some function $f_{1}(\mathrm{t})$. In mathematical terms:

$$
L^{*}\left[f_{1}(t)\right]=\frac{1}{2 z \cdot c_{2}} \cdot\left\{\begin{array}{l}
\frac{r}{c_{2}} \cdot L^{*}\left[\frac{d^{2}}{d t^{2}}\left\{\varphi(\tau) \cdot \sqrt{\tau^{2}-\varepsilon} \cdot H\left\langle t_{1}\right\rangle\right\}\right]+ \\
-\left(\frac{r}{2 c_{2}}\right)^{3} \cdot L^{*}\left[\frac{d^{4}}{d t^{4}}\left\{\varphi(\tau) \cdot\left(\tau^{2}-\varepsilon\right)^{3 / 2} \cdot H\left\langle t_{1}\right\rangle\right\}\right]
\end{array}\right\}
$$

from where it follows that:

$$
f_{1}(t)=\frac{1}{2 z \cdot c_{2}} \cdot\left[\frac{r}{c_{2}} \cdot \frac{d^{2}}{d t^{2}} F(t)-\left(\frac{r}{2 c_{2}}\right)^{3} \cdot \frac{d^{4}}{d t^{4}} G(t)\right]
$$

where

$$
F(t)=\varphi(\tau) \cdot \sqrt{\tau^{2}-\varepsilon} \cdot H\left\langle t_{1}\right\rangle
$$

and

$$
G(t)=\varphi(\tau) \cdot\left(\tau^{2}-\varepsilon\right)^{3 / 2} \cdot H\left\langle t_{1}\right\rangle .
$$

Now, as previously discussed, one has:

$$
\frac{\varepsilon}{\tau^{2}}=\frac{1}{\left(\frac{t}{z / c_{1}}\right)^{2}}<1
$$

Therefore, the function $\phi(\tau)$ is given approximately by the expression:

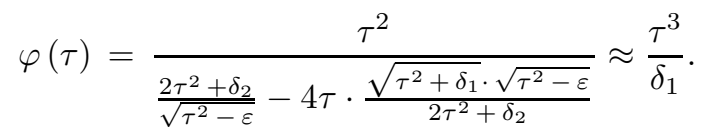


Similarly, the other components of the functions $F(t)$ and $G(t)$ are given approximately by the following expressions:

$$
\varphi(\tau) \cdot \sqrt{\tau^{2}-\varepsilon} \approx \frac{\tau^{4}}{\delta_{1}}
$$

and

$$
\varphi(\tau) \cdot\left(\tau^{2}-\varepsilon\right)^{3 / 2} \approx \frac{\tau^{6}}{\delta_{1}}
$$

Inserting Eqs (38)-(40) in Eqs (36a)-(36c), one gets the following expression for the function $f_{1}(t)$, after carrying out the derivatives and some algebraic manipulation:

$$
f_{1}(t)=\frac{q^{3} \cdot \tau^{2}}{2 r^{2} \cdot \delta_{1}} \cdot Q\left(q, \frac{\tau}{b}, t_{1}\right)
$$

where

$$
\begin{aligned}
Q\left(q, \frac{\tau}{b}, t_{1}\right)= & 12\left(1-3.75 q^{2}\right) \cdot H\left(t_{1}\right)+8 \frac{\tau}{b} \cdot\left(1-7.5 q^{2}\right) \cdot \delta\left(t_{1}\right) \\
& +\frac{\tau^{2}}{b^{2}}\left(1-22.5 q^{2}\right) \cdot \delta^{\prime}\left(t_{1}\right)-3 \frac{\tau^{3}}{b^{3}} \cdot q^{2} \cdot \delta^{\prime \prime}\left(t_{1}\right)-\frac{1}{8} \cdot \frac{\tau^{4}}{b^{4}} \cdot q^{2} \cdot \delta^{\prime \prime \prime}\left(t_{1}\right),
\end{aligned}
$$

$b=c_{2} / z, q=r / z$, and $\delta(), \delta^{\prime}(), \delta^{\prime \prime}(), \delta^{\prime \prime \prime}()$ are the Dirac delta function and its generalized derivatives with respect to time, in ascending order [11].

Let us consider the improper integral $I_{2}$ in Eq. (29b) next. By virtue of Eq. (27c), for a fixed value of the Laplace variable $\mathrm{p}$, the Hankel variable $\xi$ may be regarded as a function of $\beta$. And a change of variables defined by the transformation $\beta \cdot z=p \cdot t$ maps integral $I_{2}$ into the time domain:

$$
I_{2}=-\frac{p}{z \cdot c_{2}} \cdot \int_{0}^{\infty} \eta(\tau) \cdot J_{1}\left(k \cdot r \cdot \sqrt{\tau^{2}-1}\right) \cdot H\left\langle t_{2}\right\rangle \cdot e^{-p \cdot t} d t,
$$

with

$$
\eta(\tau)=\frac{\tau \cdot \sqrt{\tau^{2}-\delta_{1}} \cdot \sqrt{\tau^{2}-1} \cdot\left(2 \tau^{2}-1\right)}{\left(2 \tau^{2}-1\right)^{2}-4 \tau \cdot \sqrt{\tau^{2}-\delta_{1}} \cdot\left(\tau^{2}-1\right)}
$$

and $t_{2}=t-z / c_{2}$.

Proceeding as before with $I_{1}$, we now obtain integral $I_{2}$ as the Laplace transform of some function $f_{2}(\mathrm{t})$ given by:

$$
I_{2}=L^{*}\left[f_{2}(t)\right]=-\frac{1}{2 z \cdot c_{2}} \cdot\left\{\begin{array}{l}
\frac{r}{c_{2}} \cdot L^{*}\left[\frac{d^{2}}{d t^{2}}\left\{\eta(\tau) \cdot \sqrt{\tau^{2}-1} \cdot H\left\langle t_{2}\right\rangle\right\}\right]+ \\
-\left(\frac{r}{2 c_{2}}\right)^{3} \cdot L^{*}\left[\frac{d^{4}}{d t^{4}}\left\{\eta(\tau) \cdot\left(\tau^{2}-1\right)^{3 / 2} \cdot H\left\langle t_{2}\right\rangle\right\}\right]
\end{array}\right\},
$$

from where it follows that:

$$
f_{2}(t)=-\frac{q^{3}}{2 r^{2}} \cdot\left[\frac{1}{b^{2}} \cdot \frac{d^{2}}{d t^{2}} M(t)-\frac{1}{q} \cdot\left(\frac{q}{2 b}\right)^{3} \cdot \frac{d^{4}}{d t^{4}} N(t)\right],
$$

where

$$
M(t)=\eta(\tau) \cdot \sqrt{\tau^{2}-1} \cdot H\left\langle t_{2}\right\rangle
$$

and

$$
N(t)=\eta(\tau) \cdot\left(\tau^{2}-1\right)^{3 / 2} \cdot H\left\langle t_{2}\right\rangle .
$$

These equations can be simplified using the fact that: 


$$
\frac{1}{\tau^{2}}<\frac{1}{\left(\frac{t}{z / c_{1}}\right)^{2}}<1 .
$$

In this case, the function $\eta(\tau)$ and the other components of the functions $M(t)$ and $N(t)$ are given approximately by the following expressions:

$$
\begin{aligned}
& \eta(\tau)=\frac{\tau}{\frac{2 \tau^{2}-1}{\sqrt{\tau^{2}-\delta_{1}} \cdot \sqrt{\tau^{2}-1}}-4 \tau \cdot \frac{\sqrt{\tau^{2}-1}}{2 \tau^{2}-1}} \approx \frac{\tau^{3}}{\delta_{1}} \\
& \eta(\tau) \cdot \sqrt{\tau^{2}-1} \approx \frac{\tau^{4}}{\delta_{1}} \\
& \eta(\tau) \cdot\left(\tau^{2}-1\right)^{3 / 2} \approx \frac{\tau^{6}}{\delta_{1}}
\end{aligned}
$$

Inserting Eqs (46)-(48) in Eqs (44a)-(44c), one gets for function $\mathrm{f}_{2}(t)$ an expression analogous to that for function $f_{1}(t)$ in Eq. (41a). In fact, after carrying out the derivatives and some algebraic manipulation, one obtains:

$$
f_{2}(t)=-\frac{q^{3} \cdot \tau^{2}}{2 r^{2} \cdot \delta_{1}} \cdot Q\left(q, \frac{\tau}{b}, t_{2}\right)
$$

Equations (28) and (29a)-(29b) give the solution for the shear stress field under the suddenly applied concentrated unit load as:

$$
S_{r z}(r, z, t)=\frac{1}{\pi} \cdot\left(f_{1}(t)+f_{2}(t)\right)=\frac{q^{3} \cdot \tau^{2}}{2 \pi \cdot r^{2} \cdot \delta_{1}} \cdot\left[Q\left(q, \frac{\tau}{b}, t_{1}\right)-Q\left(q, \frac{\tau}{b}, t_{2}\right)\right]
$$

This is the indicial admittance function for the shear stress field.

\subsection{Unit-impulse response function}

The unit-impulse response function for the system is given by the time derivative of the indicial admittance function in Eq. (50). Applying the chain rule, one has:

$$
\begin{aligned}
h_{r z}(r, z, t) & =\left(\frac{d}{d \tau} S_{r z}\right) \cdot \frac{d \tau}{d t}=b \cdot\left(\frac{d}{d \tau} S_{r z}\right) \\
& =\frac{b}{\pi} \cdot \frac{d}{d \tau}\left(f_{1}+f_{2}\right) \\
& =\frac{q^{3} \cdot \tau}{2 \pi \cdot r^{2} \cdot \delta_{1}} \cdot\left[h\left(q, b, \tau, t_{1}\right)-h\left(q, b, \tau, t_{2}\right)\right]
\end{aligned}
$$

where

$$
\begin{aligned}
h\left(q, b, \tau, t_{1}\right)= & 24 b\left(1-3.75 q^{2}\right) \cdot H\left\langle t_{1}\right\rangle+36 \tau\left(1-6.25 q^{2}\right) \cdot \delta\left(t_{1}\right)+ \\
& +12 \frac{\tau^{2}}{b}\left(1-12.5 q^{2}\right) \cdot \delta^{\prime}\left(t_{1}\right)+\frac{\tau^{3}}{b^{2}} \cdot\left(1-37.5 q^{2}\right) \cdot \delta^{\prime \prime}\left(t_{1}\right)+ \\
& -3.75 \frac{q^{2} \cdot \tau^{4}}{b^{3}} \cdot \delta^{\prime \prime \prime}\left(t_{1}\right)-\frac{1}{8} \cdot \frac{q^{2} \cdot \tau^{5}}{b^{4}} \cdot \delta^{I V}\left(t_{1}\right) .
\end{aligned}
$$

Consequently, the dynamic shear stress field generated in the medium by a concentrated force from an explosive source at the surface with general time variation specified by the function $\Phi(t)$ is given by the convolution integral [7, 15]: 


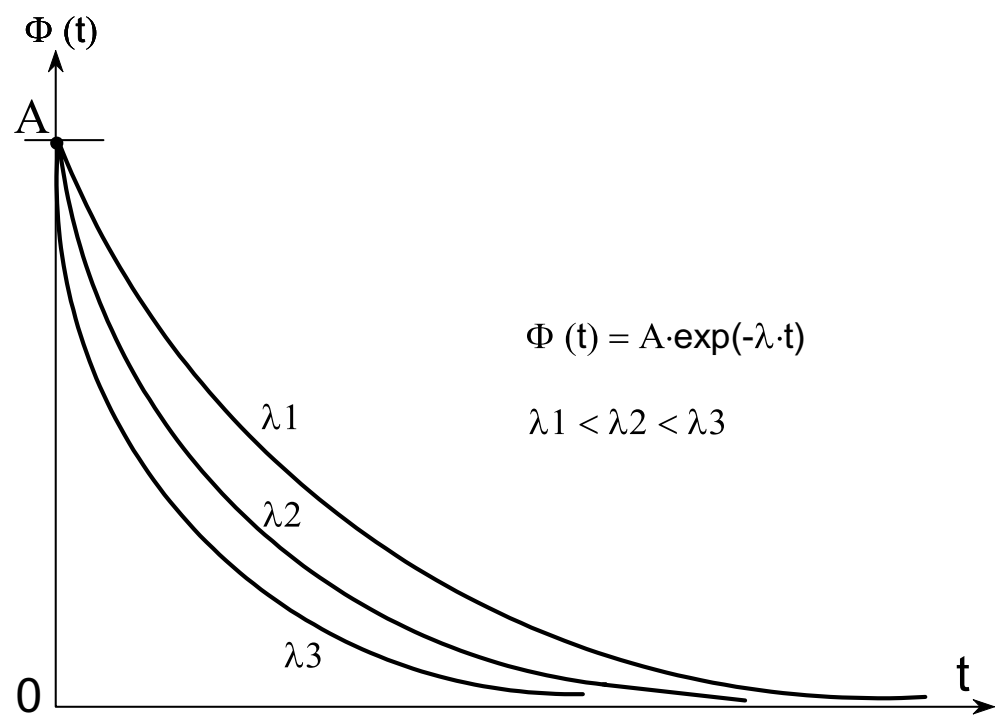

Fig. 2. Three realizations of an exponentially-decaying loading function of magnitude $\mathrm{A}$ and parameter $\lambda$, with $\lambda<\lambda_{2}<\lambda_{3}$.

$$
\begin{aligned}
\tau_{r z}(r, z, t) & =\int_{0}^{t} h_{r z}(r, z, \theta) \cdot \Phi(t-\theta) d \theta \\
& =\int_{0}^{t} \Phi(\theta) \cdot h_{r z}(r, z, t-\theta) d \theta .
\end{aligned}
$$

\subsection{Response to forcing function}

Assuming a specific time variation for the forcing function generated by the concentrated charge at the surface, the analytical solution for the shear stress field in the elastic medium can be carried out further. An exponentially decaying load (a two-parameter formulation) is postulated in this model as given by (Fig. 2):

$$
\Phi(t)=A \cdot e^{-\lambda \cdot t}
$$

Inserting Eq. (54) into the first of the Eq (53), and evaluating the ensuing integrals, the following shear stress field results:

$$
\tau_{r z}=\frac{A \cdot b^{2} \cdot q}{2 \pi \cdot \delta_{1} \cdot z^{2}} \cdot\left\{\begin{array}{c}
24 i_{1} \cdot\left(1-3.75 q^{2}\right)+36 i_{2} \cdot\left(1-6.25 q^{2}\right)+ \\
+12 i_{3} \cdot\left(1-12.5 q^{2}\right)+i_{4} \cdot\left(1-37.5 q^{2}\right)+ \\
-3.75 i_{5} \cdot q^{2}-\frac{1}{8} \cdot i_{6} \cdot q^{2}
\end{array}\right\}
$$

in which

$$
i_{k}=I_{k}\left(\frac{z}{c_{1}}\right)-I_{k}\left(\frac{z}{c_{2}}\right), \quad k=1,2, \ldots 6,
$$

and the $I_{k}$ functions are defined by: 


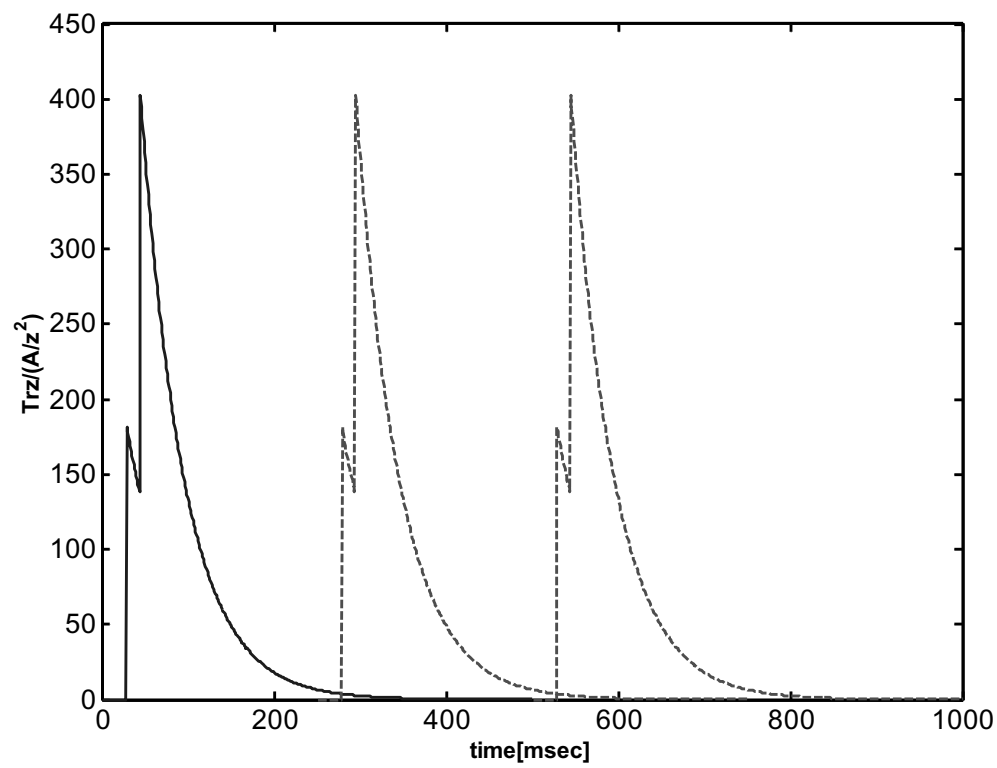

Fig. 3. Typical time history of the normalized shear stress at a field point within the linear-elastic half-space domain under a train of three sequential load pulses.

$$
\begin{aligned}
& I_{1}(\xi)=\frac{\xi^{2}}{\lambda \xi} \cdot\left\{\left(\frac{t}{\xi}-1\right)+\left(1-\frac{1}{\lambda \xi}\right) \cdot\left(1-e^{-\lambda(t-\xi)}\right)\right\} \\
& I_{2}(\xi)=\xi^{2} \cdot e^{-\lambda(t-\xi)} \\
& I_{3}(\xi)=\xi^{2} \cdot(3+\lambda \xi) \cdot e^{-\lambda(t-\xi)} \\
& I_{4}(\xi)=\xi^{2} \cdot(2+\lambda \xi) \cdot(6+\lambda \xi) \cdot e^{-\lambda(t-\xi)}, \\
& I_{5}(\xi)=\xi^{2} \cdot\left[2 \lambda \xi \cdot(5+\lambda \xi)+(3+\lambda \xi) \cdot\left(20+10 \lambda \xi+\lambda^{2} \xi^{2}\right)\right] \cdot e^{-\lambda \cdot(t-\xi)},
\end{aligned}
$$

and

$$
I_{6}(\xi)=\xi^{2} \cdot\left[3 \lambda \xi \cdot\left(30+12 \lambda \xi+\lambda^{2} \xi^{2}\right)+(3+\lambda \xi) \cdot\left(120+90 \lambda \xi+18 \lambda^{2} \xi^{2}+\lambda^{3} \xi^{3}\right)\right] \cdot e^{-\lambda(t-\xi)} .
$$

The solid curve in Fig. 3 shows in non-dimensional form a typical time history of shear stress at a field point within the linear-elastic half-space domain under a single load pulse, as predicted by Eq. (55). The soil is postulated with specific gravity $\mathrm{G}_{s}=2.65$, shear modulus of elasticity $\mathrm{G}=49.0 \mathrm{MPa}$, Poisson's ratio $\nu=0.17$, and water content $\mathrm{w}$ $=30 \%$. The field point under consideration is located at a depth $z=6 \mathrm{~m}$ and at an equal radial distance $\mathrm{r}=6 \mathrm{~m}$ from the cylindrical z-axis. Notice in the curve that the dilatational wave arrives $28 \mathrm{msec}$ after the detonation, whereas the distortional wave arrives $44 \mathrm{msec}$ after the detonation. They both contribute to the shear stress response at the point. The discontinuous curves in the figure correspond to a pair of sequential load pulses applied subsequently approximately at the same source point, for a train of three pulses.

Notice that for a fixed value of the depth $z$, there is a value of the ratio $q=r / z$ which maximizes the magnitude of the shear stress given by Eq. (55). Therefore, various specific layers can be examined successively, and the critical point experiencing the maximum shear stress level can be identified in each. In fact, the critical point for a given layer $(\mathrm{z})$ is defined by the equation for the extremum:

$$
\frac{\partial \tau_{r z}}{\partial r}=\frac{\partial \tau_{r z}}{\partial q} \cdot \frac{d q}{d r}=\frac{1}{z} \cdot \frac{\partial \tau_{r z}}{\partial q}=0 \quad \Leftrightarrow \quad \frac{\partial \tau_{r z}}{\partial q}=0 .
$$

When this condition is applied to Eq. (55), the critical location for the layer is obtained as: 


$$
r_{0}=z \cdot q_{0}=z \cdot \sqrt{\frac{24 i_{1}+36 i_{2}+12 i_{3}+i_{4}}{270 i_{1}+675 i_{2}+450 i_{3}+112.5 i_{4}+11.25 i_{5}+3 / 8 \cdot i_{6}}} .
$$

As a final step in the process of assessing the load effect, analytical expressions will be evaluated at the time of first arrival of the dilatational waves, i.e., at $t=z / c_{1}$. Again, after this time, progressive decay in the time history of response takes effect. Then, for $t=z / c_{1}$, Eq. (55) gives the shear stress field as:

$$
\tau_{r z}=\frac{A \cdot q^{3}}{2 \pi \cdot r^{2} \cdot \delta_{1}} \cdot \mathrm{X}\left(q^{2}, \varsigma_{1}, \varsigma_{2}\right),
$$

where

$$
\mathrm{X}\left(q^{2}, \varsigma_{1}, \varsigma_{2}\right)=\frac{e^{-\varsigma_{1}}}{\varsigma_{2}^{2}} \cdot\left\{\begin{array}{c}
24\left(1-3.75 q^{2}\right) \cdot\left(\varsigma_{2}-1\right) \cdot e^{\varsigma_{2}}+36\left(1-6.25 q^{2}\right) \cdot \Delta_{2}+ \\
+12\left(1-12.5 q^{2}\right) \cdot \Delta_{3}+\left(1-37.5 q^{2}\right) \cdot \Delta_{4}+ \\
-3.75 q^{2} \cdot \Delta_{5}-\frac{1}{8} \cdot q^{2} \cdot \Delta_{6}
\end{array}\right\},
$$

$\Delta_{2}=\varsigma_{1}^{2} \cdot e^{\varsigma_{1}}-\varsigma_{2}^{2} \cdot e^{\varsigma_{2}}$

$\Delta_{3}=\varsigma_{1}^{2} \cdot\left(3+\varsigma_{1}\right) \cdot e^{\varsigma_{1}}-\varsigma_{2}^{2} \cdot\left(3+\varsigma_{2}\right) \cdot e^{\varsigma_{2}}$,

$\Delta_{4}=\varsigma_{1}^{2} \cdot\left(2+\varsigma_{1}\right) \cdot\left(6+\varsigma_{1}\right) \cdot e^{\varsigma_{1}}-\varsigma_{2}^{2} \cdot\left(2+\varsigma_{2}\right) \cdot\left(6+\varsigma_{1}\right) \cdot e^{\varsigma_{2}}$,

$\Delta_{5}=\varsigma_{1}^{2} \cdot\left[2 \varsigma_{1} \cdot\left(5+\varsigma_{1}\right)+\left(3+\varsigma_{1}\right) \cdot\left(20+10 \varsigma_{1}+\varsigma_{1}^{2}\right)\right] \cdot e^{\varsigma_{1}}+$

$$
-\varsigma_{2}^{2} \cdot\left[2 \varsigma_{2} \cdot\left(5+\varsigma_{2}\right)+\left(3+\varsigma_{2}\right) \cdot\left(20+10 \varsigma_{2}+\varsigma_{2}^{2}\right)\right] \cdot e^{\varsigma_{2}},
$$

$\Delta_{6}=\varsigma_{1}^{2} \cdot\left[3 \varsigma_{1} \cdot\left(30+12 \varsigma_{1}+\varsigma_{1}^{2}\right)+\left(3+\varsigma_{1}\right) \cdot\left(120+90 \varsigma_{1}+18 \varsigma_{1}^{2}+\varsigma_{1}^{3}\right)\right] \cdot e^{\varsigma_{1}}+$

$$
-\varsigma_{2}^{2} \cdot\left[3 \varsigma_{2} \cdot\left(30+12 \varsigma_{2}+\varsigma_{2}^{2}\right)+\left(3+\varsigma_{2}\right) \cdot\left(120+90 \varsigma_{2}+18 \varsigma_{2}^{2}+\varsigma_{2}^{3}\right)\right] \cdot e^{\varsigma_{2}},
$$

and

$$
\varsigma_{1}=\frac{\lambda \cdot z}{c_{1}} ; \quad \varsigma_{2}=\frac{\lambda \cdot z}{c_{2}} .
$$

\section{Liquefaction criterion}

The semi-empirical liquefaction criterion proposed by Seed and Idriss [16] is adopted in this model on account of its simplicity and for having its fundamentals in experimental evidence under dynamic excitation. The non-zero components of stress in the sand samples that provided the experimental data for the model inference are analogous to those components of stress under blast as expressed by Eqs 11(a)-11(e). According to this criterion, a local region of a saturated soil deposit experiences liquefaction when the exciting cyclic stress ratio reaches a limit state. The cyclic shear stress ratio is defined as the fraction of the average cyclic shear stress to the static effective normal stress on the region $\left(p_{0}\right)$. The limit state of the cyclic shear stress ratio is determined from laboratory experiments. Using the data from Ishihara and Koga [13], the mean critical cyclic shear stress ratio $(y)$ depends on the number of cycles experienced by the soil deposit $(N)$ and may be summarized analytically by the following expression:

$$
y=0.45 C_{r} \cdot(N-1)^{-0.1845},
$$

where $C_{r}=$ factor modifying test results from a tri-axial test apparatus. Values of $C_{r}$ may be taken approximately as: 


$$
C_{r}= \begin{cases}0.57, & \text { for } K_{0}=0.4 \\ 0.90, & \text { for } K_{0}=1.0,\end{cases}
$$

in which $K_{0}$ is the lateral earth pressure coefficient.

Consequently, in this model it is assumed that liquefaction occurs when the load effect exceeds the soil deposit resistance, i.e.:

$$
s=\frac{\tau_{r z, \max }}{p_{0}}>y .
$$

\section{Probability of liquefaction}

In search for realistic mathematical tractability, the inherent uncertainty of physical quantities in this study is idealized next. Two separate cases will be considered corresponding to different states of uncertainty. In both cases, the explosive charge and the resistance of the soil deposit to liquefaction are considered random. In case 1, both are log-normally distributed on account of their non-negativeness. However, only the magnitude of the explosion load $(A)$ is unknown, and the analyst has knowledge of the time-decay constant $(\lambda)$, which is estimated from past observations in the field. In this case, the probability of liquefaction is given by [8]:

$$
\operatorname{Pr} o b(\text { liquefaction })=\Psi\left(-\frac{\log \left(\frac{\mu_{y}}{\mu_{s}}\right)}{\sqrt{C_{y}^{2}+C_{s}^{2}}}\right),
$$

where $\Psi$ is the standard normal distribution, log is natural logarithm, and $\left(\mu, C^{2}\right)$ is the pair (mean, square coefficient of variation) for the random variable specified by the attached subscript.

After factoring out the load magnitude, the shear stress field in Eq. (60) may be written as:

$$
\tau \equiv \tau_{r z}=A \cdot \Lambda(\lambda) .
$$

Therefore, the mean value of the shear stress in given by [15]:

$$
\mu_{\tau}=E(A) \cdot E(\Lambda)=\mu_{A} \cdot \mu_{\Lambda},
$$

where $E()$ is the mathematical expectation operator, whereas the variance may be computed approximately as:

$$
\sigma_{\tau}^{2} \approx \mu_{A} \cdot \mu_{\Lambda} \cdot\left(C_{A}^{2}+C_{\Lambda}^{2}\right) .
$$

The second-moment characterization of the load effect, i.e., the cyclic shear stress ratio is given by the mean:

$$
\mu_{s}=\frac{\mu_{\tau}}{p_{0}}
$$

and the variance:

$$
\sigma_{s}^{2}=\frac{\sigma_{\tau}^{2}}{\left(p_{0}\right)^{2}} .
$$

Ultimately, the reliability index may be computed from:

$$
\beta=\frac{\mu_{y}-\mu_{s}}{\sqrt{\sigma_{y}^{2}+\sigma_{s}^{2}}},
$$

where the second-moment characterization of the liquefaction resistance $y$ is given by the pair (mean $=\mu_{y}$, variance $\left.=\sigma_{y}^{2}\right)$ and is assumed known.

If it can be assumed that both random variables $s$ and $y$ are Gaussian, then the probability of liquefaction may be obtained as:

$$
\operatorname{Pr} o b(\text { liquefaction })=\Psi(-\beta)=1-\Psi(\beta) .
$$


If the random variables $s$ and $y$ cannot be assumed Gaussian, then Eq. (72) still provides a good estimate of the probability of liquefaction for a given situation [9].

What remains to resolve is the evaluation of the moments of the random function $\Lambda(\lambda)$ in terms of the mean and the variance of $\lambda, \mu_{\lambda}$ and $\sigma_{\lambda}^{2}$, respectively, which are given. The means of $\varsigma_{1}$ and $\varsigma_{2}$ are given by:

$$
\bar{\varsigma}_{1}=E\left(\varsigma_{1}\right)=\frac{\sqrt{\varepsilon}}{b} \cdot \mu_{\lambda} \quad \text { and } \quad \bar{\varsigma}_{2}=E\left(\varsigma_{2}\right)=\frac{1}{b} \cdot \mu_{\lambda} .
$$

Using Eq. (61c), the mean of $\Lambda$ may be computed approximately as:

$$
\mu_{\lambda}=E(\Lambda) \approx \Lambda\left(\mu_{\lambda}\right)=\Lambda\left(q^{2}, \bar{\varsigma}_{1}, \bar{\varsigma}_{2}\right),
$$

and, using Eqs (60) and (73), the variance of $\Lambda$ may be computed approximately as:

$$
\sigma_{\Lambda}^{2} \approx\left(\left.\frac{d \Lambda}{d \lambda}\right|_{\lambda=\mu_{\lambda}}\right)^{2} \cdot \sigma_{\lambda}^{2}
$$

in which

$$
\left.\frac{d \Lambda}{d \lambda}\right|_{\lambda=\mu_{\lambda}}=\frac{q^{3}}{2 \pi r^{2} \cdot \delta_{1}} \cdot\left\{\left.\frac{d \mathrm{X}}{d \varsigma_{1}}\right|_{\left(\bar{\varsigma}_{1}, \bar{\varsigma}_{2}\right)} \cdot \frac{\sqrt{\varepsilon}}{b}+\left.\frac{d \mathrm{X}}{d \varsigma_{2}}\right|_{\left(\bar{\varsigma}_{1}, \bar{\varsigma}_{2}\right)} \cdot \frac{1}{b}\right\} .
$$

\section{Parametric study}

A parametric study was conducted to examine the sensitivity of the probability of liquefaction of a given saturated sand deposit subjected to a concentrated blast force at the surface to the variation in the moments of the exponentialdecay load parameters. The following information was used as required. For the sand: specific gravity of solids, $\mathrm{G}_{s}=2.65$; elastic modulus in shear, $\mathrm{G}=49.0 \mathrm{MPa}$; Poisson's ratio, $\nu=0.17$; water content, $\mathrm{w}=30 \%$. For the explosive charge: mean magnitude of charge, $\mu_{A}=500 \mathrm{~kg}$ TNT; coefficient of variation of the magnitude of charge, C.O.V. $\cdot_{A}=1.0$; mean time-decay constant, $\mu_{\lambda}=2.0 \mathrm{sec}^{-1}$; coefficient of variation of the time-decay constant, C.O.V. $\cdot \lambda=0.2$. Liquefaction resistance: number of shear stress cycles, $\mathrm{N}=3$; coefficient of variation of the number of shear stress cycles, C.O.V. $._{N}=0.3$.

The mean and the coefficient of variation of the load magnitude and of the time-decay constant are varied over the corresponding expected range in Figs 4-9. In these figures, the depth of a location within the sand deposit has been normalized with respect to the reference depth $\mathrm{z}_{0}=18 \mathrm{~m}$, beyond which the medium response is negligible. Generally, the probability of liquefaction increases substantially with the mean load magnitude (Fig. 4) and with the load impulse (Fig. 5). In other words, for a given mean load magnitude, the more concentrated the time variation of the load, the larger the chances of liquefaction survival. Also, the probability of liquefaction increases with the dispersion of the load magnitude (coefficient of variation) for intermediate depths (6 to $15 \mathrm{~m}$ ) and, conversely, the probability of liquefaction decreases with increases in the dispersion of the load magnitude for small depths $(<6 \mathrm{~m})$. At a depth of about $6 \mathrm{~m}$, the reliability becomes zero (mean load effect and mean resistance coincide), i.e., the probability of liquefaction survival is about $50 \%$ (Fig. 6). These conclusions may also be derived about the variation of the probability of liquefaction with the dispersion of the load time-decay constant (Fig. 7).

The shear stress ratio transmitted to a given layer clearly decreases with depth (Fig. 8). The shear stress ratio (load effect) transmitted to a layer at a given depth increases with the mean load magnitude and with the load impulse. However, the shear stress ratio is relatively insensitive to the dispersion in the load magnitude. In general, as a deeper layer in the deposit is examined, the critical point for liquefaction moves away from the central vertical axis $(r=0)$ in a nonlinear fashion (Fig. 9). The location of the critical point for liquefaction $(r)$ in a given layer $(z)$ is very sensitive to the mean load time-decay constant, but this variation tends to decrease as the mean load time-decay constant increases (i.e., the $r$-versus- $z$ curve becomes flatter). 


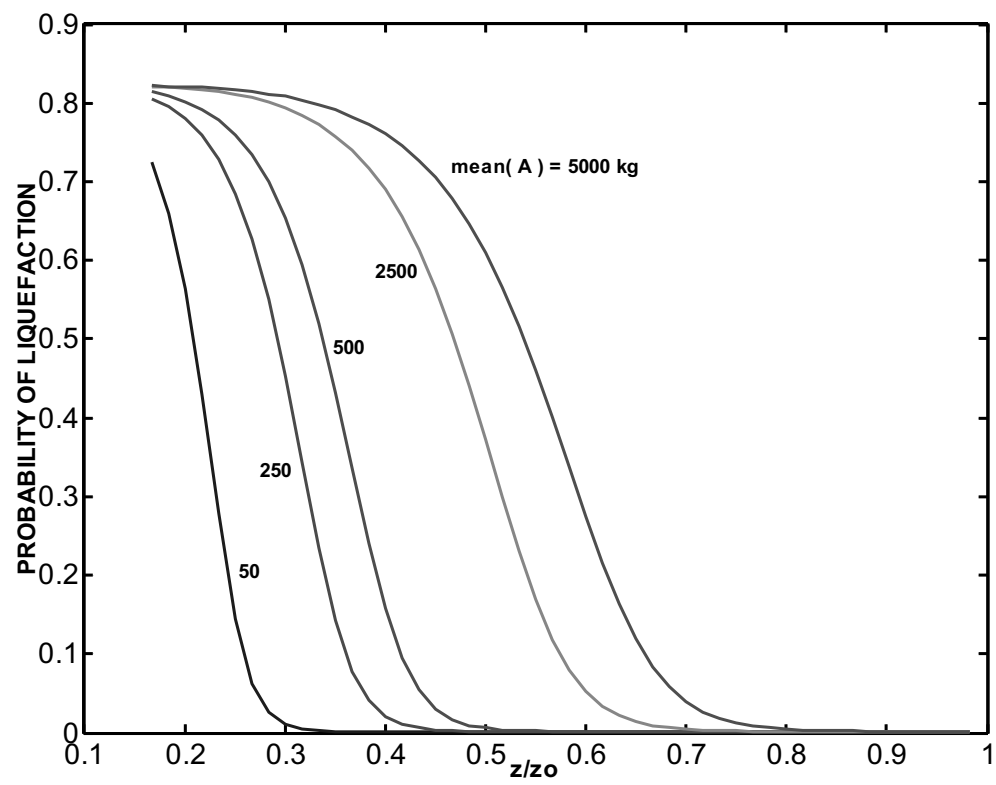

Fig. 4. Variation of the probability of liquefaction with the normalized depth (for several values of the mean load magnitude).

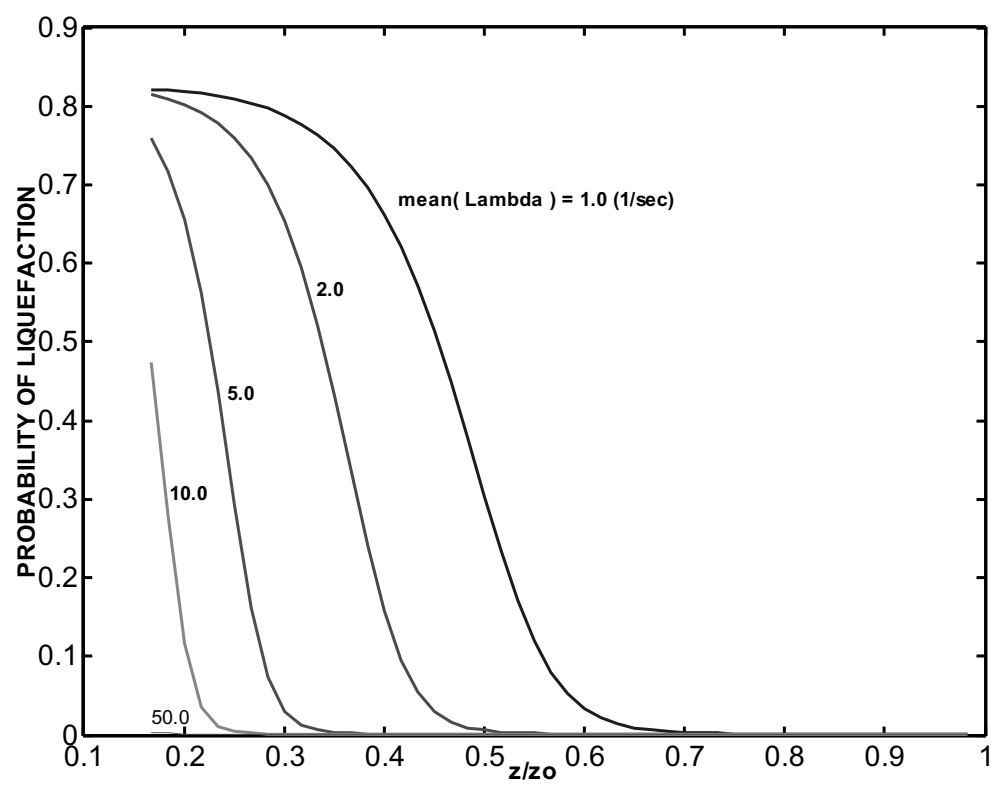

Fig. 5. Variation of the probability of liquefaction with the normalized depth (for several values of the mean time-decay constant of the applied load).

\section{Conclusions}

An engineering model for the evaluation of the probability of liquefaction of saturated sands under stress waves from a train of concentrated surface blast pulses has been formulated in this study. The model is presented in closed form within the limitations imposed by the approximate reduction of mathematical expressions involving small quantities. However, the model only represents an approximation to real blast-induced soil liquefaction, since 


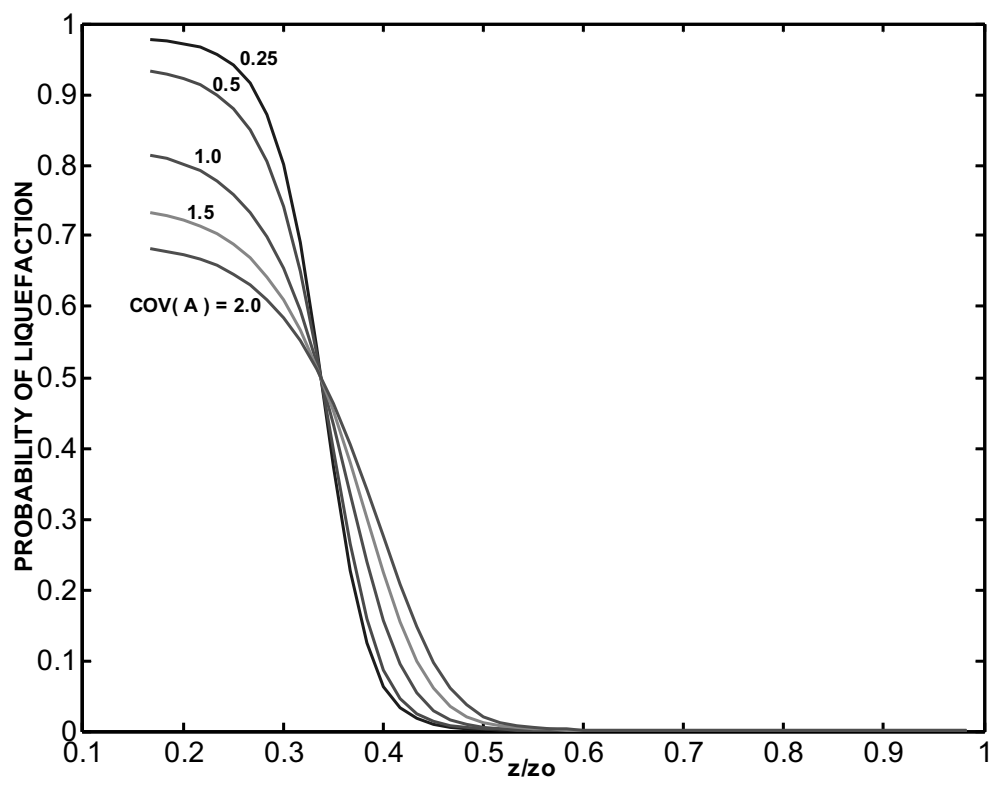

Fig. 6. Variation of the probability of liquefaction with the normalized depth (for several values of the coefficient of variation of the load magnitude).

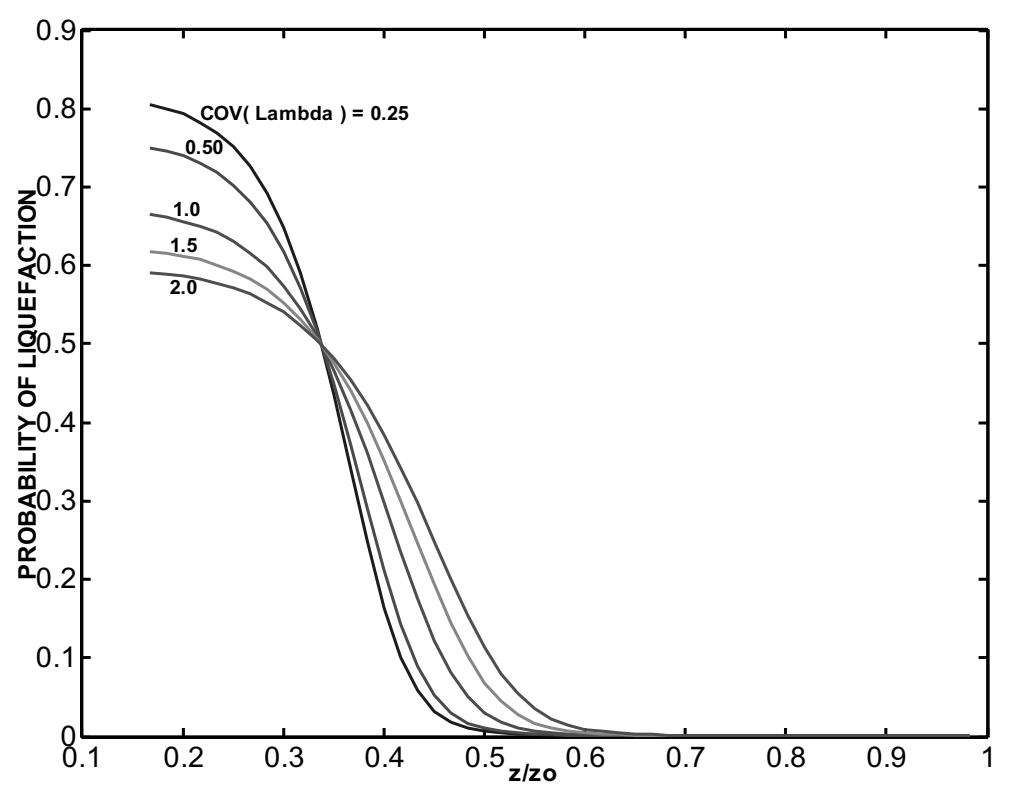

Fig. 7. Variation of the probability of liquefaction with the normalized depth (for several values of the coefficient of variation of the time-decay constant of the applied load).

necessary simplifications for the intervening physical components were postulated to allow mathematical tractability of the problem.

The liquefaction criterion adopted in this investigation was inferred from experimental data for samples of sand under non-zero components of stress analogous to those components of stress under blast in this model.

Parametric studies reveal the general increase in the probability of liquefaction with the mean load magnitude and with the impulse of the blasting load. For intermediate depths, an increase in the dispersion of the temporally- 


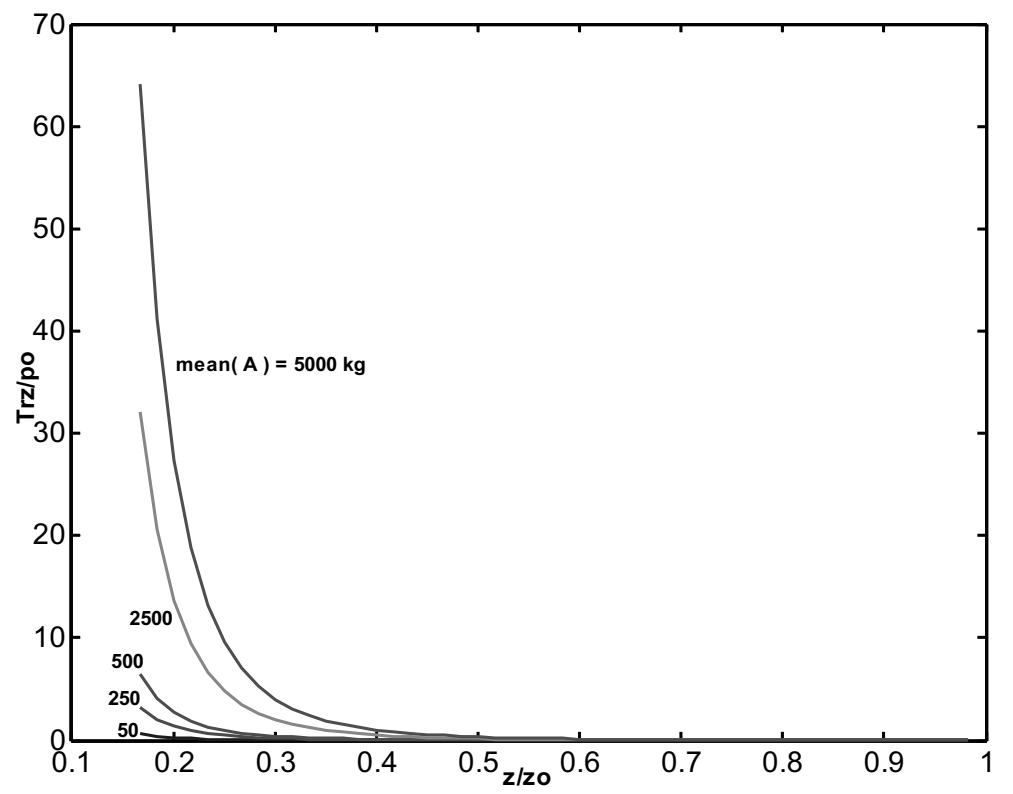

Fig. 8. Variation of the normalized oscillating shear stress (with respect to the static normal stress) with the normalized depth, for several values of the mean load magnitude.

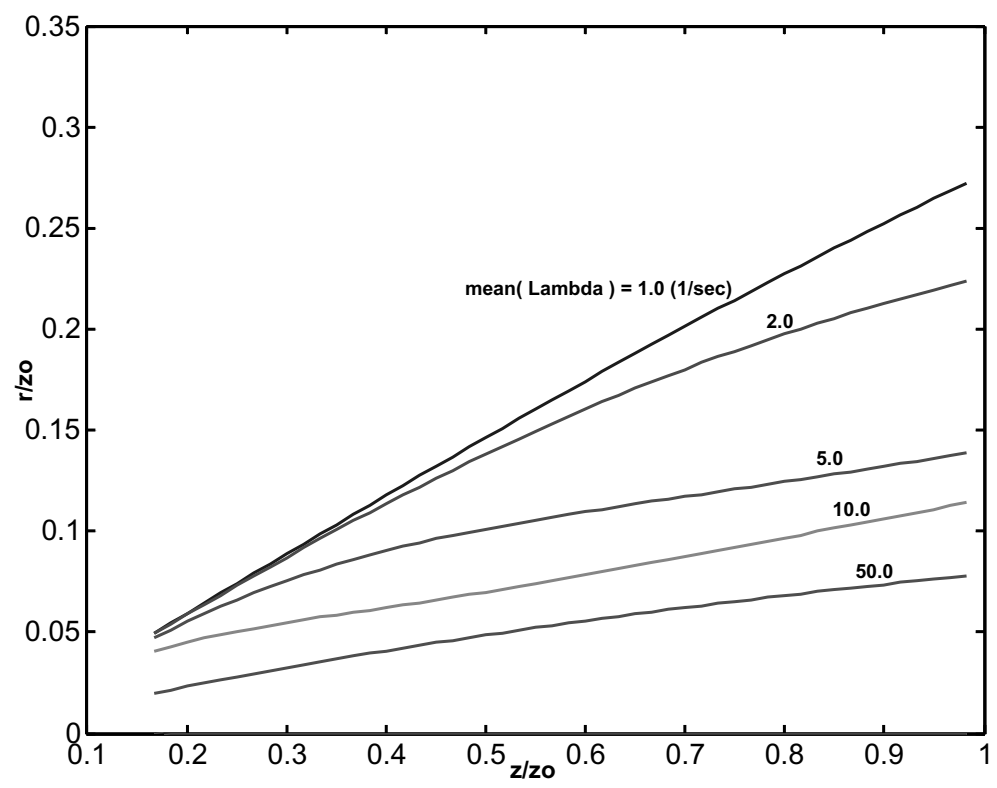

Fig. 9. Variation of the normalized radial location of the critical point for liquefaction with the normalized depth (for several values of the mean time-decay constant of the applied load).

exponential-load parameters in this investigation also increases the probability of liquefaction.

\section{Acknowledgments}

This investigation was sponsored by the US Department of the Army, Corps of Engineers, Headquarters, under the Asymmetric Terrorist Threat Reduction Research Program. The author gratefully acknowledges this support. 
Permission to publish was granted by the Director of the Geotechnical and Structures Laboratory of the US Army Engineer Research and Development Center. Approved for public release; distribution is unlimited.

\section{References}

[1] J.R. Benjamin and C.A. Cornell, Probability, Statistics, and Decision for Civil Engineers, McGraw-Hill, New York, NY, 1970.

[2] J.M. Bolton, D.S. Durnford and W.A. Charlie, One-dimensional Shock and Quasi-static Liquefaction of Silt and Sand, ASCE, Journal of Geotechnical Engineering 120(10) (1994), 1874-1889.

[3] W.A. Charlie and D.O. Doehring, Groundwater Table Mounding, Pore Pressure, and Liquefaction Induced by Explosions: Energy-Distance Relations, Reviews of Geophysics, 45, RG4006, Paper No. 2006RG000205, 2007.

[4] W.A. Charlie, G.E. Veyera, D.S. Durnford and D.O. Doehring, Porewater Pressure Increases in Soil and Rock from Underground Chemical and Nuclear Explosions, International Journal of Engineering Geology 43(12) (1996), 225-236.

[5] W.A. Charlie, Review of Present Practices Used in Predicting the Effects of Blasting on Pore Pressure, US Department of the Interior, Bureau of Reclamation, Engineering and Research Center, Technical Report GR-85-9, Denver, CO, 1985.

[6] W.A. Charlie, J. Shinn and S. Melzer, Blast Induced Soil Liquefaction, Proceedings of the 2nd US National Conference on Earthquake Engineering, Stanford, CA, 1979.

[7] R.W. Clough and J. Penzien, Dynamics of Structures, McGraw-Hill, New York, NY, 2004.

[8] S.-H. Dai and M.-O. Wang, Reliability Analysis in Engineering Applications, Van Nostrand Reinhold, New York, NY, 1992.

[9] O. Ditlevsen, Generalized Second-moment Reliability Index, Journal of Structural Mechanics 7(4) (1979), 435-451.

[10] K.F. Graff, Wave Motion in Elastic Solids, Universities Press (for Ohio State University Press), Belfast, Northern Ireland, 1975.

[11] M.D. Greenberg, Foundations of Applied Mathematics, Prentice-Hall, Englewood Cliffs, NJ, 1978.

[12] I.M. Idriss and R.W. Boulanger, Soil Liquefaction during Earthquakes, Earthquake Engineering Research Institute Monograph, Berkeley, CA. 2008.

[13] K. Ishihara and Y. Koga, Case Studies of Liquefaction in the 1964 Niigata Earthquake, Japanese Society of Soil Mechanics and Foundation Engineering, Journal of Soils and Foundations 21(3) (1981), 35-52.

[14] H. Kolsky, Stress Waves in Solids, Oxford University Press, New York, NY, 1953.

[15] H.J. Larson and B.O. Shubert, Probabilistic Methods in Engineering Sciences, Volume I: Random Variables and Stochastic Processes, John Wiley, New York, NY, 1979.

[16] H.B. Seed and I.M. Idriss, Ground Motions and Soil Liquefaction During Earthquakes, Earthquake Engineering Research Institute Monograph, Berkeley, CA, 1982. 

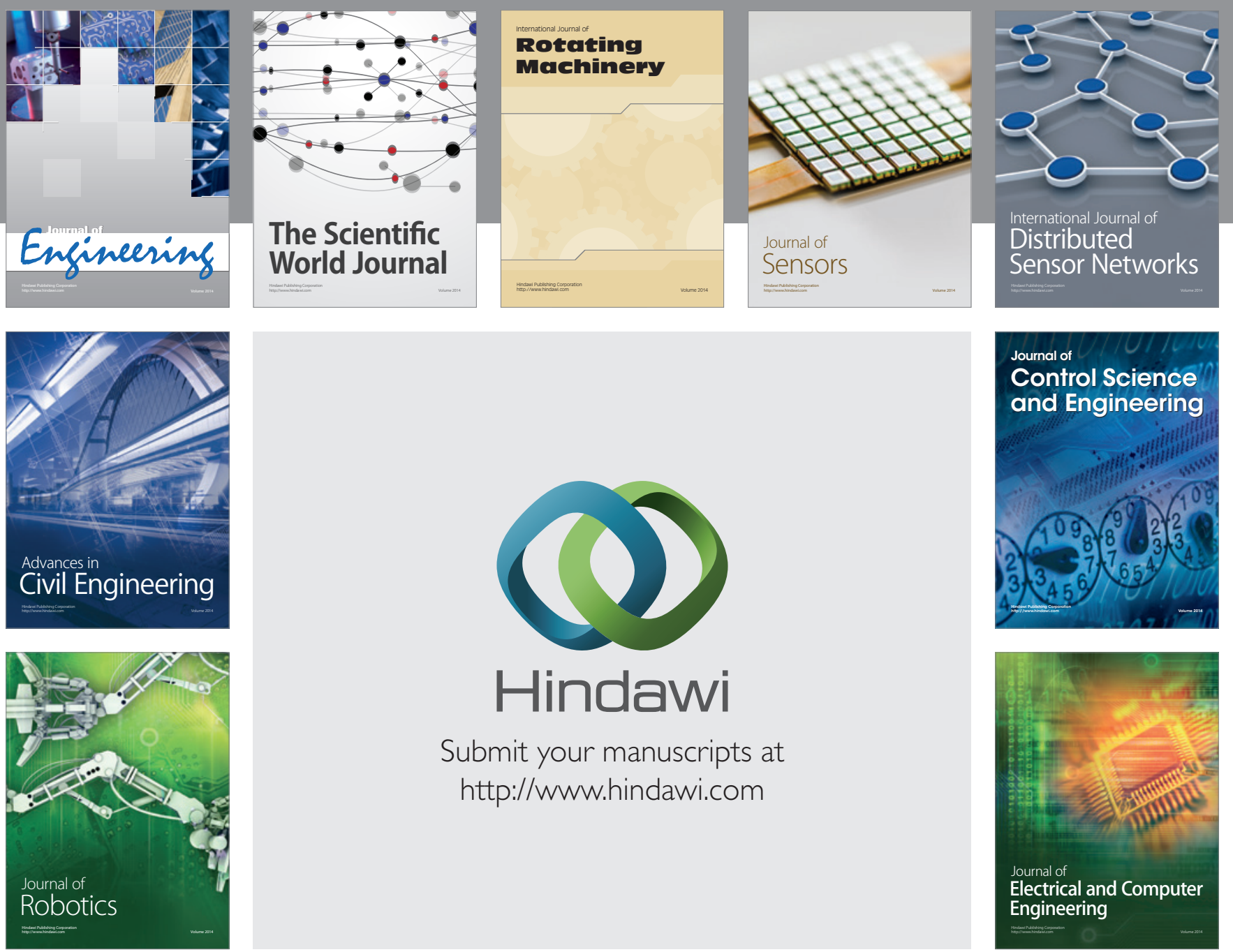

Submit your manuscripts at

http://www.hindawi.com
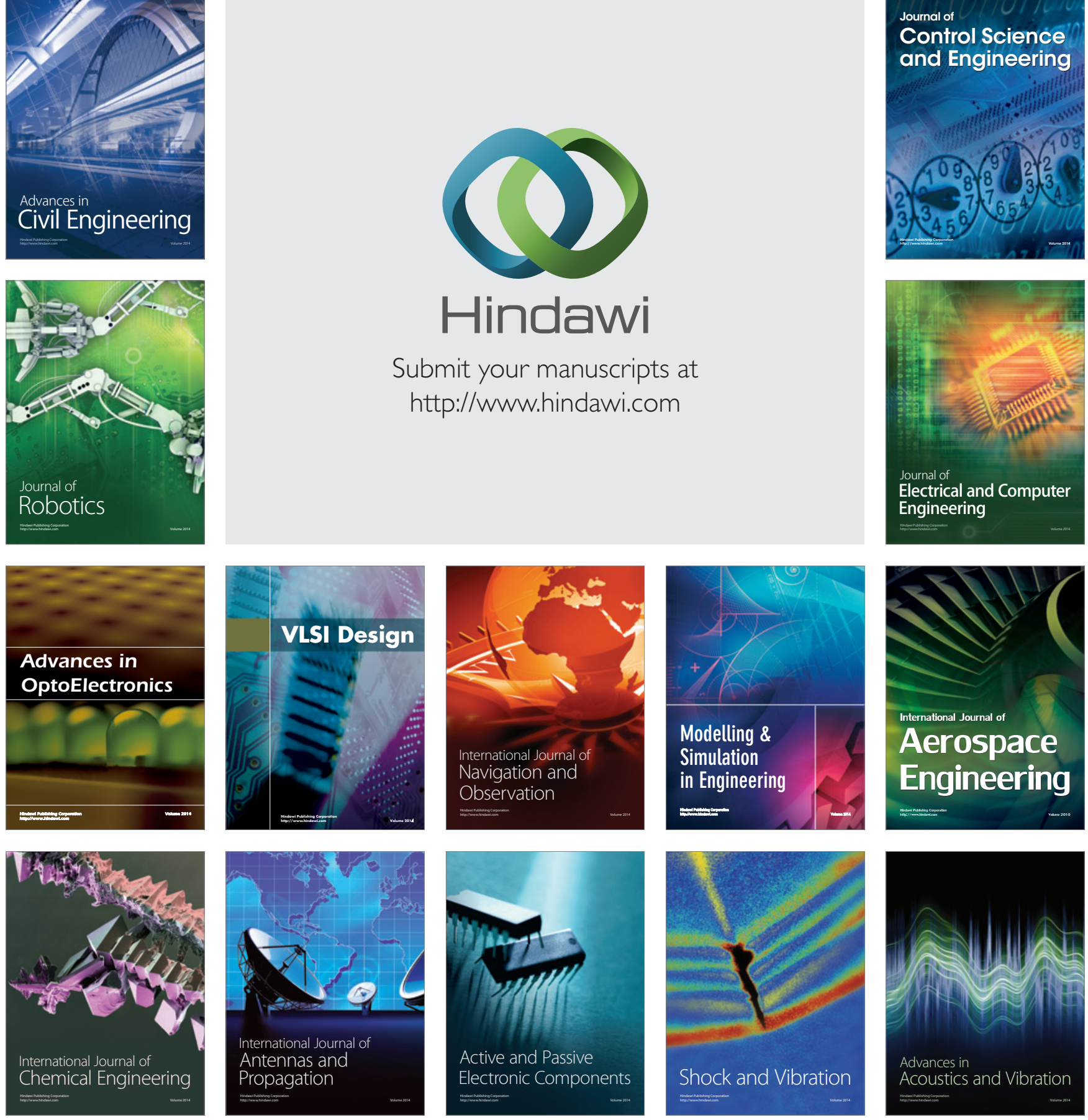